\title{
Exploring gluon saturation with photons in high-energy hadron-hadron collisions
}

\section{Sanjin Benić*†}

Yukawa Institute for Theoretical Physics, Kyoto University, Kyoto 606-8502, Japan

E-mail: benic.sanjin@yukawa.kyoto-u.ac.jp

\section{Kenji Fukushima}

Department of Physics, The University of Tokyo, 7-3-1 Hongo, Bunkyo-ku, Tokyo

113-0033, Japan

E-mail: fuku@nt.phys.s.u-tokyo.ac.jp

\section{Oscar Garcia-Montero}

Institut für Theoretische Physik, Universität Heidelberg, Philosophenweg 16, 69120

Heidelberg, Germany

E-mail: garcia@thphys.uni-heidelberg.de

\section{Raju Venugopalan}

Physics Department, Brookhaven National Laboratory, Bldg. 510A, Upton, NY 11973, USA

E-mail: rajuv@mac.com

We numerically compute the inclusive photon production in $\mathrm{p}+\mathrm{p}$ collisions within the Color Glass Condensate framework as determined through the valence $q g \rightarrow q \gamma$ leadingorder channel and the $g g \rightarrow q \bar{q} \gamma$ next-to-leading-order channel, respectively. We find the $g g \rightarrow q \bar{q} \gamma$ channel accounts for more than $90 \%$ of the cross section at the center of mass energies of $\sqrt{s}=7 \mathrm{TeV}$ and $13 \mathrm{TeV}$. The obtained numerical results compare well with the ATLAS and CMS $\mathrm{p}+\mathrm{p}$ data for inclusive isolated photon production within the region where $x<0.01$ in either of the colliding protons.

XXVI International Workshop on Deep-Inelastic Scattering and Related Subjects (DIS2018) 16-20 April 2018

Kobe, Japan

\footnotetext{
*Speaker.

${ }^{\dagger}$ On leave of absence from Department of Physics, Faculty of Science, University of Zagreb, Bijenička c. 32, 10000 Zagreb, Croatia. 


\section{Introduction}

We are motivated by the large gluon occupations at the LHC and its potential to explore the physics of gluon saturation. The inclusive photon production cross section was measured in $\mathrm{p}+\mathrm{p}$ collisions by ATLAS $[1](\sqrt{s}=7 \mathrm{TeV})$ and CMS $[2,3,4](\sqrt{s}=2.76 \mathrm{TeV}$ and $7 \mathrm{TeV}$ ) up to photon rapidity $\left|\eta_{\gamma}\right| \leq 2.5$, partially covering also the region $x<0.01$ where deviations to the photon spectrum from the collinear perturbative QCD results, and due to the effect of saturation of gluons, are expected. The latter is described by the Color Glass Condensate (CGC) effective field theory (EFT) wherein the gluon distributions become transverse momentum dependent and strongly modified in the infrared, at around the saturation scale $Q_{S}$.

We have used the CGC EFT and applied it to inclusive photon production: $\mathrm{p}+\mathrm{p} \rightarrow$ $\gamma+X$. We used the dilute-dense framework where all-order rescatterings on the dense target, together with its non-linear quantum evolution effects, are taken into account, so that the forward collision region probes the small- $x$ content of the target proton.

The leading-order (LO) contribution comes from the $q \rightarrow q \gamma$ channel (in the target background). The main formula for the inclusive cross section is given as $[5,6,7,8]$ (with recent applications in [9])

$$
\begin{aligned}
& \frac{\mathrm{d} \sigma^{\mathrm{LO}}}{\mathrm{d}^{2} \boldsymbol{k}_{\gamma \perp} \mathrm{d} \eta_{\gamma}}=S_{\perp} \sum_{f} \frac{\alpha_{e} q_{f}^{2}}{16 \pi^{2}} \int_{\boldsymbol{q}_{\perp}} \int_{x_{p, \min }}^{1} \mathrm{~d} x_{p} f_{q, f}^{\mathrm{val}}\left(x_{p}, Q^{2}\right) \tilde{\mathcal{N}}_{t, Y_{t}}\left(\boldsymbol{q}_{\perp}+\boldsymbol{k}_{\gamma \perp}\right) \\
& \times \frac{1}{q^{+} l^{+}}\left\{-4 m_{f}^{2}\left[\frac{l^{+2}}{\left(q \cdot k_{\gamma}\right)^{2}}+\frac{q^{+2}}{\left(l \cdot k_{\gamma}\right)^{2}}+\frac{k_{\gamma}^{+2}}{\left(l \cdot k_{\gamma}\right)\left(q \cdot k_{\gamma}\right)}\right]\right. \\
& \left.+4\left(l^{+2}+q^{+2}\right)\left[\frac{l \cdot q}{\left(l \cdot k_{\gamma}\right)\left(q \cdot k_{\gamma}\right)}+\frac{1}{q \cdot k_{\gamma}}-\frac{1}{l \cdot k_{\gamma}}\right]\right\},
\end{aligned}
$$

where $f_{q, f}^{\mathrm{val}}\left(x_{p}, Q^{2}\right)$ is the valence quark distribution so that the sum runs over the valence $f=u, d$ flavors only. For consistency reasons we are taking only the valence content at LO, whereas the sea quark contribution is taken into account by the $g \rightarrow q \bar{q} \gamma$ NLO channel to be discussed below. The distribution of gluons in the target is described by a fundamental Wilson line dipole $\tilde{\mathcal{N}}_{t, Y_{t}}\left(\boldsymbol{k}_{\perp}\right)$. For further details see also [10].

At NLO we take into account the $g \rightarrow q \bar{q} \gamma$ [11] channel as dominating the mid-rapidity region over the remaining $q \rightarrow q g \gamma$ [12] and $g \rightarrow q^{*} \bar{q}^{*} \rightarrow \gamma$ [13] channels also present at this order. The inclusive cross section is given as [11] (see also [10])

$$
\begin{aligned}
\frac{\mathrm{d} \sigma^{\mathrm{NLO}}}{\mathrm{d}^{2} \boldsymbol{k}_{\gamma \perp} \mathrm{d} \eta_{\gamma}}= & S_{\perp} \sum_{f} \frac{\alpha_{e} \alpha_{S} N_{c}^{2} q_{f}^{2}}{64 \pi^{4}\left(N_{c}^{2}-1\right)} \int_{\eta_{q} \eta_{p}} \int_{\boldsymbol{q}_{\perp} \boldsymbol{p}_{\perp} \boldsymbol{k}_{1 \perp} \boldsymbol{k}_{\perp}} \frac{\varphi_{p}\left(Y_{p}, \boldsymbol{k}_{1 \perp}\right)}{\boldsymbol{k}_{1 \perp}^{2}} \tilde{\mathcal{N}}_{t, Y_{t}}\left(\boldsymbol{k}_{\perp}\right) \tilde{\mathcal{N}}_{t, Y_{t}}\left(\boldsymbol{P}_{\perp}-\boldsymbol{k}_{1 \perp}-\boldsymbol{k}_{\perp}\right) \\
& \times\left[2 \tau_{g, g}\left(\boldsymbol{k}_{1 \perp} ; \boldsymbol{k}_{1 \perp}\right)+4 \tau_{g, q \bar{q}}\left(\boldsymbol{k}_{1 \perp} ; \boldsymbol{k}_{\perp}, \boldsymbol{k}_{1 \perp}\right)+2 \tau_{q \bar{q}, q \bar{q}}\left(\boldsymbol{k}_{\perp}, \boldsymbol{k}_{1 \perp} ; \boldsymbol{k}_{\perp}, \boldsymbol{k}_{1 \perp}\right)\right],
\end{aligned}
$$

at large $N_{c}$. Here $\varphi_{p}\left(Y_{p}, \boldsymbol{k}_{1 \perp}\right)$ is the unintegrated gluon distribution (UGD) in the projectile proton and the sum $\sum_{f}$ runs over $f=u, d, s, c, b$ flavors in our computation. 


\section{Calculation setup and numerical results}

For the parton distribution functions (PDFs) we use the central CTEQ6M set [14]. The gluon UGD of the proton is fixed by its relation to the adjoint dipole: $\varphi_{p}\left(Y_{p}, \boldsymbol{k}_{1 \perp}\right)=$ $S_{\perp} N_{c} \boldsymbol{k}_{1 \perp}^{2} \mathcal{N}_{p, Y_{p}}\left(\boldsymbol{k}_{1 \perp}\right) / 4 \alpha_{S}$. The gluon dipoles are evolved according to the running coupling Balistsky-Kovchegov equation (rcBK) [15, 16], with the initial condition set by the McLerran-Venugopalan (MV) model [17] at $x=0.01$ that provides a good description of the $J / \Psi$ production in $\mathrm{p}+\mathrm{p}$ [18]. The region $x>0.01$ is fixed by the matching to the gluon $\mathrm{PDF}$ as in [18]. For the quark masses we took $m_{u}=m_{d}=0.005 \mathrm{GeV}, m_{s}=0.095 \mathrm{GeV}$, $m_{c}=1.3 \mathrm{GeV}$ and $m_{b}=4.5 \mathrm{GeV}$. We have varied their values according to their current experimental uncertainties leading to about $10 \%$ deviations in the cross section that is taken into account as a part of our systematics.

We calculate the spectrum of isolated photons that is obtained by applying an isolation cone $R$ as

$$
\theta\left(\sqrt{\left(\eta_{\gamma}-\eta\right)^{2}+\left(\phi_{\gamma}-\phi\right)^{2}}-R\right)
$$

with $\eta$ and $\phi$ being the rapidity and the azimuthal angle of the quark. This constraint suppresses collinear emissions within the hadron jets such as decay photons but also fragmentation photons.
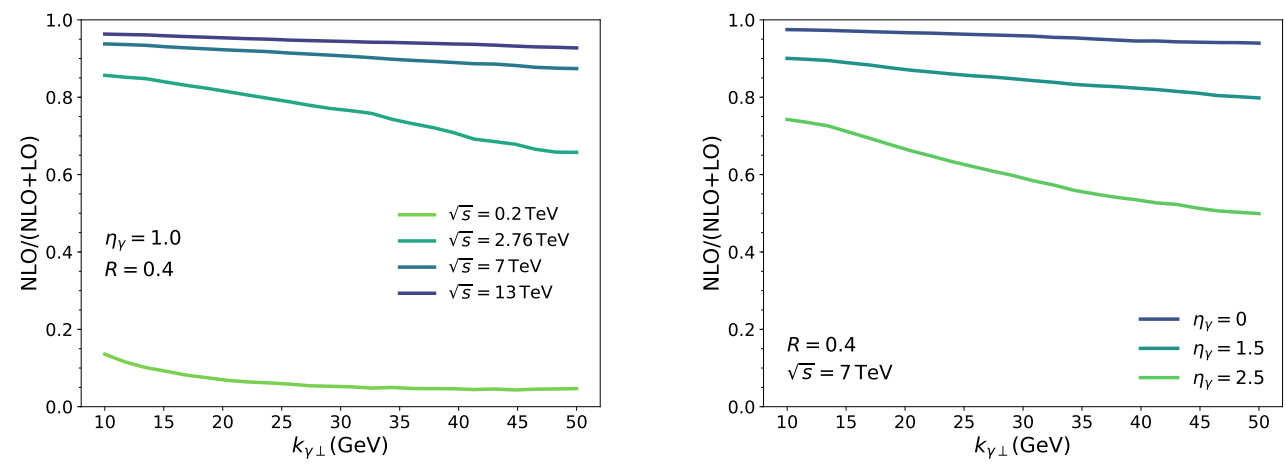

Figure 1: Fraction of the inclusive photon cross section from the NLO $g g \rightarrow q \bar{q} \gamma$ channel relative to the total $\mathrm{NLO}+\mathrm{LO}$ contribution, as a function of $k_{\gamma \perp}$ for different collision energies (left panel) and different $\eta_{\gamma}$ (right panel).

In the following we show the numerical results based on the LO and NLO contributions described in the previous section and compare with the relevant experimental data. The LHC data from CMS and ATLAS account for photons with $k_{\gamma \perp} \sim 20 \mathrm{GeV}$. We have numerically established [10] that at such transverse momenta the full-CGC formula (1.2) is already well approximated by its $k_{\perp}$-factorized form ${ }^{1}$

$$
\begin{aligned}
\frac{\mathrm{d} \sigma_{k_{\perp} \text {-fact }}^{\mathrm{NLO}}}{\mathrm{d}^{2} \boldsymbol{k}_{\gamma \perp} \mathrm{d} \eta_{\gamma}}= & S_{\perp} \sum_{f} \frac{\alpha_{e} \alpha_{S} N_{c}^{2} q_{f}^{2}}{64 \pi^{4}\left(N_{c}^{2}-1\right)} \int_{\eta_{q} \eta_{p}} \int_{\boldsymbol{q}_{\perp} \boldsymbol{p}_{\perp} \boldsymbol{k}_{1 \perp}} \frac{\varphi_{p}\left(Y_{p}, \boldsymbol{k}_{1 \perp}\right)}{\boldsymbol{k}_{1 \perp}^{2}} \mathcal{N}_{t, Y_{t}}\left(\boldsymbol{P}_{\perp}-\boldsymbol{k}_{1 \perp}\right) \\
& \times\left[2 \tau_{g, g}\left(\boldsymbol{k}_{1 \perp}\right)+\tau_{q, q}\left(\boldsymbol{k}_{1 \perp}\right)+\tau_{\bar{q}, \bar{q}}\left(\boldsymbol{k}_{1 \perp}\right)+2 \tau_{g, q}\left(\boldsymbol{k}_{1 \perp}\right)+2 \tau_{g, \bar{q}}\left(\boldsymbol{k}_{1 \perp}\right)\right] .
\end{aligned}
$$

\footnotetext{
${ }^{1} k_{\perp}$-factorization becomes broken at lower $k_{\gamma \perp}$ : at around $k_{\gamma \perp} \sim 1 \mathrm{GeV}$ the breaking is about $10 \%$, see $[10]$.
} 
With this observation the rest of the results will be presented using (2.2).

On Fig. 1 we show the fractional contribution of the NLO cross section to the full $\mathrm{LO}+\mathrm{NLO}$ result with varying the collision energy (left panel) and photon rapidity (right panel) using the isolation cut $R=0.4$. Whereas at the $\sqrt{s}=0.2 \mathrm{TeV}$ RHIC energy NLO provides a correction to the full cross section, the increase to the LHC energy marks the NLO contribution to more than $60 \%$ at $2.76 \mathrm{TeV}$ and more than $90 \%$ at $7 \mathrm{TeV}$ and $13 \mathrm{TeV}$. Similar trends are found when moving from the mid-rapidity to the forward photon rapidities.
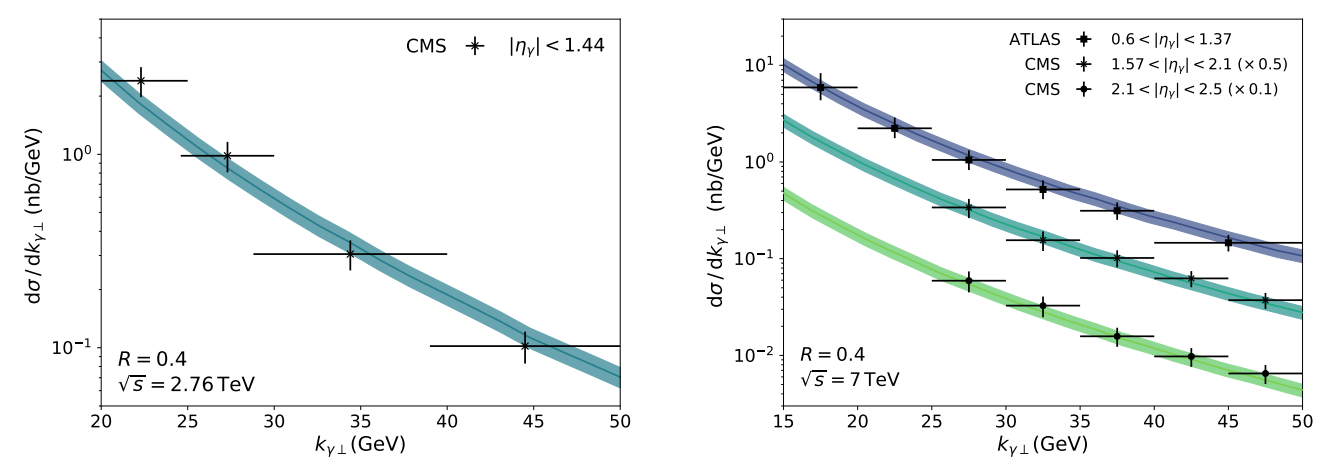

Figure 2: Comparison to the $\mathrm{p}+\mathrm{p}$ photon data at $\sqrt{s}=2.76 \mathrm{TeV}$ (left panel, CMS [2]) and $\sqrt{s}=7$ $\mathrm{TeV}$ (right panel, ATLAS [1], CMS [4] across several rapidity bins. The central lines are obtained with a $K$-factor of $K=2.4$.

The left panel on Fig. 2 gives a comparison to the CMS data at $2.76 \mathrm{TeV}$ [2] where we find a good agreement by using an overall $K$-factor of $K=2.4$. On right panel Fig. 2 we compare with the ATLAS [1] and CMS [4] data at $7 \mathrm{TeV}$; a good agreement is found using the same $K=2.4$.

\section{Conclusions}

We have for the first time numerically computed the CGC NLO $g \rightarrow q \bar{q} \gamma$ contribution to inclusive photon production $\mathrm{p}+\mathrm{p} \rightarrow \gamma X$. We have found that the NLO channel dominates by more than $90 \%$ over the LO $q g \rightarrow q \gamma$ channel at the $\sqrt{s}=7 \mathrm{TeV}$ and $13 \mathrm{TeV}$ energies. Our results demonstrate fair agreement with the available LHC data. Working still within the dilute-dense formalism our formulas are suitable for future applications of photon production in $\mathrm{p}+\mathrm{A}$ collisions where the saturation effects are expected to be more pronounced.

\section{Acknowledgments}

We thank Kevin Dusling for his help with the rcBK solutions and for providing his rcBK code. S. B. acknowledges discussions with Yoshitaka Hatta and Davor Horvatić. S. B. was supported by the European Union Seventh Framework Programme (FP7 2007-2013) under 
grant agreement No. 291823, Marie Curie FP7-PEOPLE2011-COFUND NEWFELPRO Grant No. 48. S. B. is supported by the JSPS fellowship and JSPS Grant-in-Aid for JSPS fellows 17F17323. S. B. acknowledges HRZZ Grant No. 8799 at the University of Zagreb for computational resources. This work is part of and supported by the DFG Collaborative Research Centre "SFB 1225 (ISOQUANT)".

\section{References}

[1] ATLAS collaboration, G. Aad et al., Measurement of the inclusive isolated prompt photon cross section in pp collisions at $\sqrt{s}=7$ TeV with the ATLAS detector, Phys. Rev. D83 (2011) 052005, [1012.4389].

[2] CMS collaboration, S. Chatrchyan et al., Measurement of isolated photon production in $p p$ and PbPb collisions at $\sqrt{s_{N N}}=2.76$ TeV, Phys. Lett. B710 (2012) 256-277, [1201.3093].

[3] CMS collaboration, V. Khachatryan et al., Measurement of the Isolated Prompt Photon Production Cross Section in pp Collisions at $\sqrt{s}=7$ TeV, Phys. Rev. Lett. 106 (2011) 082001, [1012.0799].

[4] CMS collaboration, S. Chatrchyan et al., Measurement of the Differential Cross Section for Isolated Prompt Photon Production in pp Collisions at 7 TeV, Phys. Rev. D84 (2011) 052011, [1108.2044].

[5] B. Z. Kopeliovich, A. V. Tarasov and A. Schafer, Bremsstrahlung of a quark propagating through a nucleus, Phys. Rev. C59 (1999) 1609-1619, [hep-ph/9808378].

[6] F. Gelis and J. Jalilian-Marian, Photon production in high-energy proton nucleus collisions, Phys. Rev. D66 (2002) 014021, [hep-ph/0205037].

[7] R. Baier, A. H. Mueller and D. Schiff, Saturation and shadowing in high-energy proton nucleus dilepton production, Nucl. Phys. A741 (2004) 358-380, [hep-ph/0403201].

[8] B. Z. Kopeliovich, H. J. Pirner, A. H. Rezaeian and I. Schmidt, Azimuthal anisotropy of direct photons, Phys. Rev. D77 (2008) 034011, [0711.3010].

[9] B. Ducloué, T. Lappi and H. Mäntysaari, Isolated photon production in proton-nucleus collisions at forward rapidity, Phys. Rev. D97 (2018) 054023, [1710.02206].

[10] S. Benić, K. Fukushima, O. Garcia-Montero and R. Venugopalan, Constraining unintegrated gluon distributions from inclusive photon production in proton-proton collisions at the LHC, 1807.03806.

[11] S. Benic, K. Fukushima, O. Garcia-Montero and R. Venugopalan, Probing gluon saturation with next-to-leading order photon production at central rapidities in proton-nucleus collisions, JHEP 01 (2017) 115, [1609.09424].

[12] T. Altinoluk, N. Armesto, A. Kovner, M. Lublinsky and E. Petreska, Soft photon and two hard jets forward production in proton-nucleus collisions, JHEP 04 (2018) 063, [1802.01398].

[13] S. Benic and K. Fukushima, Photon from the annihilation process with CGC in the $p A$ collision, Nucl. Phys. A958 (2017) 1-24, [1602.01989].

[14] J. Pumplin, D. R. Stump, J. Huston, H. L. Lai, P. M. Nadolsky and W. K. Tung, New generation of parton distributions with uncertainties from global QCD analysis, JHEP 07 (2002) 012, [hep-ph/0201195]. 
[15] I. Balitsky, Operator expansion for high-energy scattering, Nucl. Phys. B463 (1996) 99-160, [hep-ph/9509348].

[16] Y. V. Kovchegov, Small $x$ F(2) structure function of a nucleus including multiple pomeron exchanges, Phys. Rev. D60 (1999) 034008, [hep-ph/9901281].

[17] K. Dusling, F. Gelis, T. Lappi and R. Venugopalan, Long range two-particle rapidity correlations in $A+A$ collisions from high energy QCD evolution, Nucl. Phys. A836 (2010) 159-182, [0911.2720].

[18] Y.-Q. Ma and R. Venugopalan, Comprehensive Description of Production in Proton-Proton Collisions at Collider Energies, Phys. Rev. Lett. 113 (2014) 192301, [1408.4075]. 